\title{
Quantitative Estimation of Gallic Acid as Biomarker in Lipitame Tablets by HPTLC Densitometry for Diabetic Dyslipidemia
}

\author{
Sheeraz Siddiqui', Khan Usmanghani' ${ }^{1,2}$, Aqib Zahoor ${ }^{2}$, Zeeshan Ahmed Sheikh², \\ Saleha Suleman Khan'2 \\ ${ }^{1}$ Faculty of Eastern Medicine, Hamdard University, Karachi, Pakistan \\ ${ }^{2}$ Research and Development Department, Herbion Pakistan (Pvt.) Limited, Karachi, Pakistan \\ Email: ugk 2005@yahoo.com
}

Received 20 August 2014; revised 26 September 2014; accepted 11 October 2014

Copyright (C) 2014 by authors and Scientific Research Publishing Inc.

This work is licensed under the Creative Commons Attribution International License (CC BY). http://creativecommons.org/licenses/by/4.0/

(c) (i) Open Access

\begin{abstract}
Lipitame is a poly herbal formulation comprised of Terminalia arjuna, Terminalia belerica, Commiphora mukul and Phyllanthus emblica. The formulation is investigated for its analysis evaluation. Biomarkers of three herbs such as Terminalia arjuna, Terminalia belerica, and Phyllanthus emblica have been already cited to contain gallic acid, which was qualitatively and quantitatively estimated. In the present study rapid and inexpensive qualification methods for the quality control of Terminalia arjuna, Terminalia belerica, Commiphora mukul and Phyllanthus emblica on thin layer chromatography (TLC) were developed and validated. The solvent system used was toluene:ethyl acetate:formic acid:methanol (12:9:4:0.5). The scanning of plate was performed linearly at $273 \mathrm{~nm}$ (absorption) by use of a TLC Scanner III CAMAG with a deuterium source, and the area of spots corresponding to Gallic acid standard was integrated. It was found that gallic acid has been found in the Lipitame tablets on HPTLC densitometry assessment compared with authentic gallic acid reference standard.
\end{abstract}

\section{Keywords}

Lipitame, Tablets, Quantitative Estimation, Densitometry, Diabetic Dyslipidemia

\section{Introduction}

Inadequately controlled hyperglycemia has been cited as a primary cause of diabetic complications. Increased serum levels of lipids in diabetics (as observed by high levels of triglycerides, total cholesterol and LDL-cho- 
lesterol) are partially responsible for and further exacerbate the damaging effects of diabetic hyperglycemia. Because there has been no effective and inexpensive therapeutic option to control glycemic or lipidemic levels in diabetic patients; these patients often suffer severe complications, including nephropathy, retinopathy, neuropathy and atherosclerosis. Although improved glycemic control can reduce the incidence and progression of diabetic complications, implementation and monitoring of glycemic control are arduous and expensive. Many diabetic complications are believed to occur through the oxidative action of glucose. In particular, the high oxidant activity in diabetics coupled with dyslipidemia can lead to the formation of advanced glycation endproducts (AGEs). The presence of AGEs is associated with the formation of arterial atheromas and ultimately, to the development of atherosclerosis. These complications can be mitigated in part by certain antioxidants, including superoxide dismutase, catalase and gluathione. Blocking the oxidative action of glucose responsible for diabetic vascular dysfunction has been validated as one approach to reduce the occurrence of diabetic complications. For example, aldose reductase inhibitors have been shown to prevent or reduce different components of vascular dysfunction, cataract formation, neuropathy and nephropathy in animal model. Furthermore, antioxidants (such as vitamin E, vitamin C and alpha lipoic acid) and antiplatelet agents (such as aspirin and ticlopidine) are being tested to determine their efficacy against the progression of certain diabetic complications, such as non-proliferative diabetic retinopathy.

Although some strides have been made to control glycemic levels and the associated oxidant activity in diabetics, little progress has been made to address the underlying problem of diabetic dyslipidemia. By lowering levels of triglycerides, total cholesterol and LDL-cholesterol in diabetics, development or progression of atherosclerosis and other diabetic complications could be slowed or eradicated. Advantageously, such therapy could be combined with hypoglycemic medications to synergistically treat diabetic patients. Therefore, it is of great importance for the long-term quality of life for diabetic patients that therapeutic options can be made available to treat diabetic dyslipidemia.

The present study is directed to a polyherbal formulation Lipitame comprised of Terminalia arjuna, Terminalia belerica, Commiphora mukul and Phyllanthus emblica or a mixture of the active ingredients that have been extracted from such herbs. The herbal compositions of the present invention are effective for the treatment of conditions involving atherosclerosis, stress and anxiety, for use in cardioprotection, cardio-toning, and hyperlipidemia. The medicinal herbal composition such as Terminalia arjuna (Roxb.) Wight \& Arn., Terminalia belerica (Gaertn.) Roxb., Commiphora mukul (Hook ex Stock) Linn. and Phyllanthus emblica Linn. have been used in $10 \%-15 \%$ by weight of selection of herbal drugs for hyperlipidemia to formulate the dosage which is in tablet form. The Unani herbal drugs have been cited for ethnobotanaical and scientific evidences and are selected for hyperlipidemia after thorough Qarabadini and published literature from electronic journals.

\section{Material and Methods}

\subsection{Formula of Tablet Lipitame (Unani Medicine)}

The Unani medicine based tablets "Lipitame" has the following ingredients: Terminalia arjuna Bark (Arjun) powder, 1000 gm; Commiphora mukul gum resin (muqil), 1300 gm; Terminalia chebula (epicarp, harh) powder, 500 gm; Phyllanthus emblica fruit (amla) powder, $500 \mathrm{gm}$.

\subsection{Manufacturing Process}

All herbs are purchased from the local market then cleaned and examined for their impurities and adulteration. The herbs are weighted according to the formulation and three drugs respectively; Terminalia chebula, Phyllanthus emblica and Terminalia arjuna are powdered through grinding machine and passed through the sieve no 50. The powder is further checked for its homogenous consistency. Commiphora mukul is soaked in water for a night and then boiled along with continuous stirring. After boiling and cooling the solution of Commiphora mukul is filtered and the impurities are separated. This cooled filter solution of Commiphora mukul is mixed with the powdered drug to develop the granules for tablet making by passing through the sieve no 80 . The granules are mixed with binder gum acacia for half hour thoroughly in a mixer.

The granules are dried at $35^{\circ} \mathrm{C}$ for a day in oven and then these granules were used to make the compressed tablets weighing each $1 \mathrm{gm}$ on average and they were stored in air tight plastic jars. After the above testing the tablets are then send for blister allo-allo foil. Each blister contains ten tablets. Three blisters are put in a box for 
30 days dosage (recommended dose 1 tablet at bedtime with plane water).

\subsection{Testing Specification}

The tablets are checked for disintegration time which is 15 minutes for Lipitame. Hardness of tablets are checked which is $6 \mathrm{~kg}$. Friability test under limit i.e. $0.5 \%$. Moisture test is $3 \%$.

\subsection{Indications}

Primary hyperlipidemia and mixed dyslipidemia, hypertriglyceridemia.

\subsection{Dosage and Administration}

Recommended dose for Lipitame is one tablet $(1 \mathrm{gm})$ at bedtime with plane water.

\subsection{Dosage Form and Packaging}

Tablet: 1 gm, 30 tablets $(10 \times 3$ unit dose).

\subsection{Contraindication}

Active liver and renal diseases, pregnancy, nursing mothers.

\subsection{Adverse Effects}

Mostly tolerated but in certain cases mild constipation, dry mouth and mild hypotension with headache.

\section{Quantitative Estimation of Gallic Acid in Lipitame Tablet by HPTLC Densitometry}

Lipitame tablets consist of Phyllanthus emblica, Terminalia chebula, Commiphora mukul and Terminalia arjuna. On literature citation it has been found that all the three drugs contains gallic acids where as Commiphora mukul elaborate no gallic acid.

Equipment: CAMAG Scanner III, CAMAG Linomat 5 or Equivalent

TLC Plates: HPTLC silica gel $\mathrm{G}_{60} \mathrm{~F}_{254}$

Solvent system:Toluene:Ethyl acetate:Formic acid:Methanol

12:9:4:0.5

Wave length: $273 \mathrm{~nm}$

\subsection{Standard Preparation}

Prepare standard solution containing known concentration $(0.4 \mathrm{mg} / \mathrm{ml})$ by dissolving $4 \mathrm{mg}$ standard of gallic acid monohydrate in $10 \mathrm{ml}$ of methanol.

\subsection{Sample Preparation}

Weigh about $2.5 \mathrm{~g}$ of tablet powder (note exact weight) in to $100 \mathrm{ml}$ of conical flask. Add $30 \mathrm{ml}$ of methanol heat to boil on water bath for 15 minute filter the solution in $250 \mathrm{ml}$ round bottomed flask. Repeat extraction of the remaining residue of tablet powder four times more using $30 \times 4 \mathrm{ml}$ portions ( 5 times in total) as mention above. Collect methanol soluble fraction into the same round-bottomed flask. Evaporate the methanol under vacuum. Dissolve the dry residue in $5 \mathrm{ml}$ of methanol and transfer quantitatively into a $10 \mathrm{ml}$ volumetric flask. Bring the solution's volume to the mark with methanol.

\subsection{Procedure}

\subsubsection{TLC Preparation}

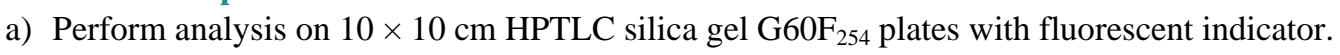

b) Before start the analysis, HPTLC plate cleans by predevelopment with methanol by ascending method. (Note: 
immerse HPTLC Plate in a CAMAG glass chamber $(20 \times 20 \mathrm{~cm})$, contains $30 \mathrm{ml}$ methanol as solvent system cover the chamber with glass lid and wait to develop the plate to the top with methanol. After complete development, remove the plate from TLC glass chamber and dry it in an oven at $105^{\circ} \mathrm{C}$ for $5 \mathrm{~min}$.)

\subsubsection{Application Procedure}

a) Apply three spots of $10 \mu \mathrm{l}$ (in the form of band) of standard preparation along with three spots of $10 \mu \mathrm{l}$ of sample preparation as the bands on the same plate by means of a CAMAG Linomat 5 (automated spray-on applicator equipped with a $100 \mu \mathrm{l}$ syringe and operated with the settings band length $6 \mathrm{~mm}$, distance between bands $14 \mathrm{~mm}$, distance from the plate side edge $15 \mathrm{~mm}$, and distance from the bottom of the plate $15 \mathrm{~mm}$ ).

b) After sample application, dry the plate in hot air oven at $105^{\circ} \mathrm{C}$ for $5 \mathrm{~min}$.

\subsubsection{TLC Development}

a) Develop the plate by immersing sample HPTLC Plate in a CAMAG glass chamber $(20 \times 20 \mathrm{~cm})$ contained the solvent system (Toluene:Ethyl acetate:Formic acid:Methanol (12:9:4:0.5)), wait to develop the plate to the distance of 8 to $9 \mathrm{~cm}$ (see Figure 1).

b) After complete development, allow the plate to dry by keeping in fume cupboard for 10 minutes and then keep in hot air oven for $5 \mathrm{~min}$ at $105^{\circ} \mathrm{C}$.

\subsubsection{TLC Scanning}

a) Scan the plate in the densitometer by linear scanning at $273 \mathrm{~nm}$ (absorption) by use of a TLC Scanner III CAMAG with a deuterium source, and integrate the area of the spots of the sample (see Figure 2) and corresponding to gallic acid standard (see Figure 3).

Calculate the amount of gallic acid in $\mathrm{mg}$ per $10 \mathrm{ml}$ in Lipitame tablet by following formula.

\subsection{Content of Gallic Acid}

$$
\frac{A_{S M P} \times W_{S T D} \times f \times \text { Dilution of Smp } \times \text { Application vol. of } S \mathrm{mp} \times P \times 0.5}{A_{S T D} \times \text { Dilution of } \operatorname{Std} \times W_{S M P} \times \text { Application of vol. } \operatorname{Std} \times 100}
$$

$A_{S M P}=$ Avg. Area of Sample;

$A_{\text {STD }}=$ Avg. Area of Standard;

$W_{S T D}=$ Weight of Standard, mg;

$W_{S M P}=$ Weight of Sample, g;

Dilution of Smp = Dilution of Sample, $\mathrm{ml}$;

Dilution of Std = Dilution of Standard, ml;

$P=$ Percent Purity of Standard;

$f(0.904)=$ conversion factor of gallic acid monohydrate in gallic acid .

Note: quantity of gallic acid in Lipitame tablet should be NLT $2.72 \mathrm{mg} / \mathrm{tablet}$.

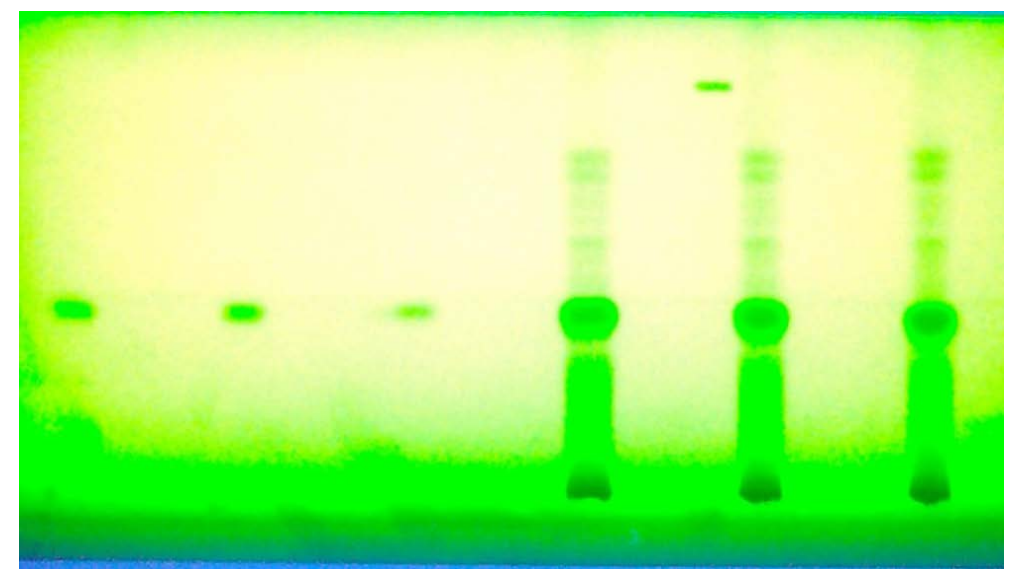

Figure 1. TLC image of gallic acid analysis in Lipitame tablet. 

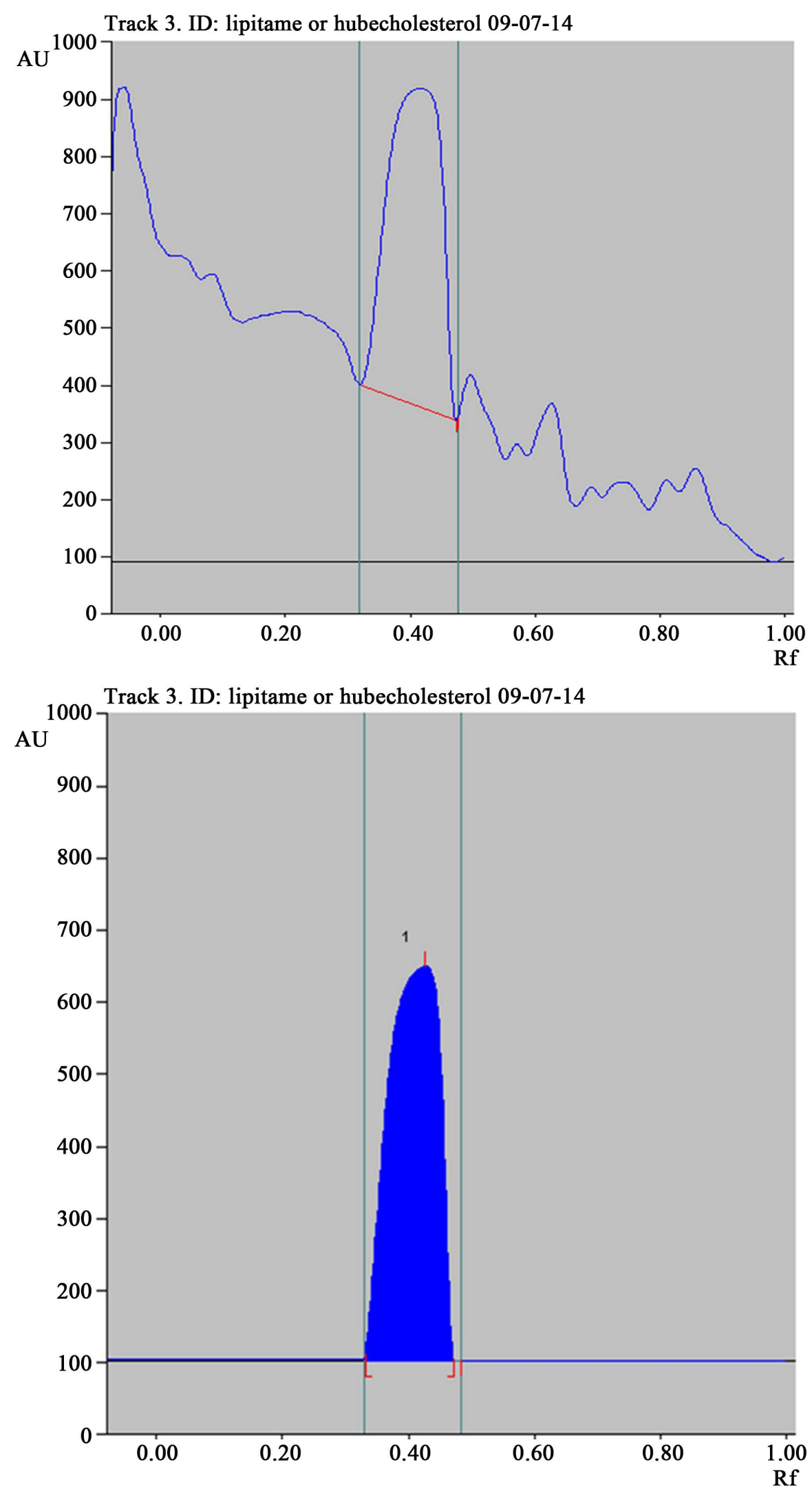

Figure 2. Peak response of gallic acid in Lipitame tablet. 

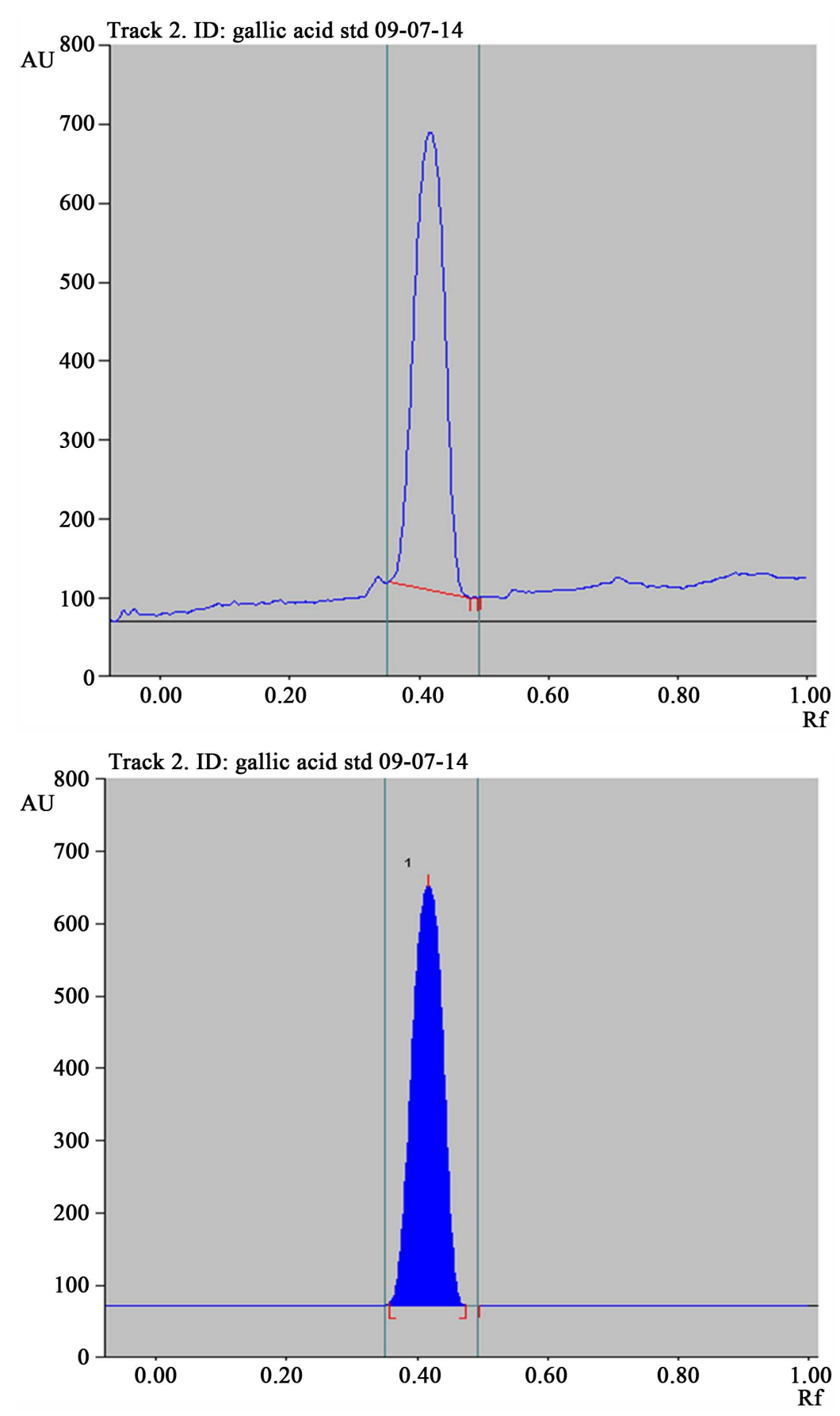

Figure 3. Peak response of gallic acid STD.

\section{Results and Discussion}

The quantity of gallic acid in Lipitame tablets is as follows:

$$
X=\frac{34847.966 \times 4.0 \times 0.904 \times 10 \times 5 \times 99 \times 0.986}{17727.866 \times 2.5454 \times 10 \times 5 \times 100}=2.72587 \mathrm{mg} / \text { tablet }
$$

The gallic acid content was also tested in a tablet containing high gallic acid (positive controls) and a tablet which did not contain gallic acid (negative controls) with the same method and comparable results were observed, confirming the quantification of gallic acid in the formulation (see Figure 4). 


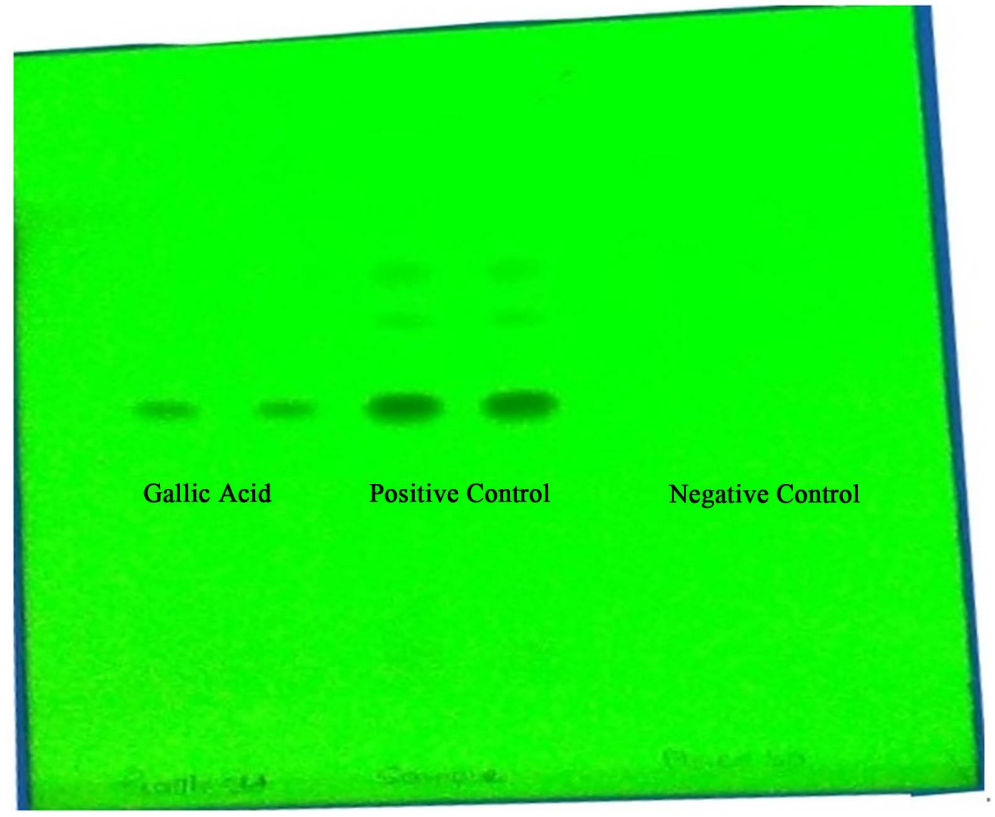

Figure 4. TLC image of Gallic acid, positive and negative control.

Standardization of specific biologically active gallic acid component was identified as material in the poly herbal formulation and quantitative analysis cover identification of chemical components where as quantitative assay measure the identification and level of bio marker in the extract establish the standard of that particular compound for validation. Lipitame is a poly herbal formulation designed by the candidate for clinical trials delineated here with in this research based study. So as to standardized the procedure of the finished tablet product and there in the four different herbal drugs such as Terminalia arjuna, Terminalia belerica, Commiphora mukul and Phyllanthus emblica. The formulation organoleptic and physic-chemical characteristics such as identification by TLC, foreign matter, total ash, acid insoluble ash, alcohol; soluble extraction and water soluble extraction are adequately presented herewith. Physico-chemical evaluation such as appearance, $\mathrm{pH}$ determination plays a significant role in the quality assessment. The identification with quantitative reaction and preparation of $0.1 \%$ alcohol solution exhibit the indication polarity concern of plant materials. TLC was done to augment the determination of gallic acid components and there in the identification was located. All these values show that the physic-chemical parameters adopted the values were within the limits and repeated experiments confirmed the verification. HPLC was performed to confirm the quantitative and qualitative presence of gallic acid for validation. The present standardization under taken reveal compliance with all the physic-chemical and analytical procedures, therefore it is concluded that Lipitame tablets is well standardized product at the base line parameters. As Lipitame consists of Terminalia arjuna, Terminalia belerica, Commiphora mukul and Phyllanthus emblica and where as all the three Terminalia arjuna, Terminalia belerica and Phyllanthus emblica contains gallic acid, therefore in the quantitative estimation gallic acid is well represented in different chromatogram. The gallic acid also inhibit the different forms of microbiological organisms so it is useful as well in diabetic dyslipidemia.

If these analytical profiles compared with the work of Modi et al. where Zeal cough syrup employing physico-chemical test and bioassay marker compounds agree with quality and potency [1]. In another study Desai and coworker validated the, manufacturing procedure of Vasu cough syrup with the poly herbal formulation for compliance for their reproducibility [2]. The work done by Khadair and associates validation analysis of hederacoside C, the marker of Ivy plant and percentage recovery was given as 99.6\% [3]. In a patent of cough syrup the extract of Adhatoda vasica along with Hedychium spp. and Curcuma spp. cough syrup has been validated in greater details [4]. Therefore, Lipitame tablets standardization fall under the specific guide lines of quality herbal medicine and in line with different as mentioned earlier follow the prerequisite for global harmonization.

The bark of Terminalia arjuna is known for its heart-health benefits in ayurvedic literature. This has been further supported by in vivo studies on animal and human volunteers. But there is no detailed study on identification of the active ingredients such as polyphenols. Polyphenols possesses antioxidant properties and are wellknown health actives, it is important to characterise polyphenols in Terminalia arjuna. Aqueous extract of Ter- 
minalia arjuna bark was analysed for its composition and molecular weight distribution by dialysis. Compositional analysis revealed that it has $44 \%$ polyphenols and dialysis study showed that $70 \%$ of the polyphenols have molecular weight greater than $3.5 \mathrm{kDa}$. High performance liquid chromatography and liquid chromatography-mass spectrometry analysis of Terminalia arjuna, confirmed that it contains flavon-3-ols such as (+)-catechin, $(+)$-gallocatechin and (-)-epigallocatechin. Phenolic acids such as gallic acid, ellagic acid and its derivatives were also found in Terminalia arjuna extract. Ellagic acid derivatives were isolated and their spectral studies indicated that isolated compounds were 3-O-methyl-ellagic acid 4-O-aD-xylopyranoside, ellagic acid and 3-O-methyl ellagic acid 3-O-rhamnoside. Hydrolysis and thiolysis studies of high molecular weight polyphenols indicated that they are proanthocyanidins. Given these results, it may be possible to attribute the heart-health effects of Terminalia arjuna to these polyphenols which may be responsible for the endothelial benefit functions like tea [5].

Phyllanthus emblica A tannin containing gallic acid, ellagic acid and glucose in its molecule and naturally present in the fruit, prevents or retard oxidation of vitamin and renders the fruit a valuable antiscorbutic activity in the fresh as well as in the dry condition. The distribution of tannin percentage in the plant is as follows: fruit 28 percentage, twig bark 21 percentage, stem bark 8 - 9 percentage, and leaves 22 percentage respectively. The fruit contains two tannins, one giving on hydrolysis gallic acid, ellagic acid and glucose and the other giving ellagic acid and glucose only [6].

The anticholesterolaemic and antiatherogenic effects of the fruits of Phyllanthus emblica have been studied. The Department of Food and Nutrition, Lady Irwin College, University of Delhi did the research over the supplementation of diet with Phyllanthus emblica in normal and hypercholesterolaemic men aged 35 - 55 years and found to be effective hypocholesterolemic [7]. Phyllanthus emblica fruit juice has been proved to be hypolipidemic at the Department of Home Science (Food and Nutrition), University of Rajasthan, Jaipur, India. The fruit juice was administered at a dose of $5 \mathrm{ml} / \mathrm{kg}$ body weight per rabbit per day for 60 days reduced serum cholesterol, triglycerides, phospholipids and LDL levels by $82 \%, 66 \%, 77 \%$ and $90 \%$ respectively [8]. Similarly, the ethanol extract of Phyllanthus emblica at a dose of $10 \mathrm{or} 20 \mathrm{mg} / \mathrm{kg}$ body weight/day for 20 days to rats fed $1 \%$ cholesterol diet significantly reduced total, free and LDL-Cholesterol levels in a dose-dependent manner [9]. Flavonoid extracts from the fruits of Phyllanthus emblica inhibited synthesis and enhanced degradation of cholesterol via increased hepatic HMG-CoA reductase [10]. In another study, the fruit juice of Phyllanthus emblica shown to reduce the oxidative stress induced by ischemic-reperfusion injury (IRI) in rat heart in three different doses 250, 500, $750 \mathrm{mg} / \mathrm{kg}$ for 30 days. These changes were due to increased production of myocardial endogenous antioxidants like superoxide dismutase (SOD), catalase and glutathione peroxidase (GPx) in rat [11]. Another study shown the dried fruit powder to be hypolipidemic in rabbits by reducing $42 \%, 29 \%$ and $31 \%$ of total cholesterol, triglycerides and LDL-Cholesterol and by increasing 33\% of HDL-Cholesterol and reducing 38\% of plaque areas in vascular system [12]. Phyllanthus emblica aqueous extract also reduces cholesterol in alloxaninduced diabetic rats along with glucose levels [13]. In another study in rabbits for 12 weeks showed increased lipid mobilization and catabolism and retarded deposition of lipids in the extrahepatic tissue [14].

Terminalia chebula fruit contains about $30 \%$ of an astringent substance called chebulinic acid, tannic acid about $20 \%$ to $40 \%$, gallic acid, resin and some purgative principle of the nature of anthraquinone [15].

Terminalia chebula has been analyzed for its antioxidant activity at different magnitudes of potency [16]. Chebulanin of Terminalia chebula has its inhibitory effects on the lipid peroxidation and the production of superoxide radicals in the body [17]. It also possessed hypocholesterolemic activity against cholesterol-induced hypercholesterolemia and atherosclerosis in rabbits [18].

In conclusion, a HPTLC method has been developed and it can be used for the quantitative determination of gallic acid in lipitame tablet. Its mean advantages are its simplicity accuracy and selectivity. This method can also be used conveniently for the estimation of gallic acid in other herbal preparations and may be utilized for standardization purpose.

\section{Conclusion}

In this study for medicinal plants component, Terminalia arjuna, Terminalia belerica, Commiphora mukul and Phyllanthus emblica were formulated for the indication of diabetic dyslipidemia activity. TLC of extract and gallic acid reference standard were carried out. The $\mathrm{R}_{f}$ value $(0.4)$ of gallic acid spot in both extract and reference standard was found comparable under UV light at $254 \mathrm{~nm}$. Quantitative and spectrophotometric estimation of gallic acid was found between the range of $0.035 \%$ and $0.07 \%$. HPLC in Liptame tablets was determined as $0.3 \mathrm{mg}$ per $10 \mathrm{ml}$. The analytical profile of Lipitame tablets and that in gallic acid have been validated to prove 
that Lipitame tablets in different batches confirmed the verification.

\section{References}

[1] Modi, J., Soni, H., Pandya, K., Patel, G. and Patel, N. (2014) A Detail Phyto-Chemical Evaluation of Herbo-Mineral Formulation Used in Respiratory Diseases. Journal of Pharmacognosy and Phytochemistry, 2, 36-42.

[2] Desai, L., Oza, J. and Khatri, K. (2012) Prospective Process Validation of Polyherbal Cough Syrup Formulation. Journal of Advanced Pharmaceutical Technology \& Research, 2, 225-231.

[3] Khdair, A., Mohammad, M.K., Tawaha, K., Al-Hamarsheh, E., AlKhatib, H.S., Al-khalidi, B., Bustanji, Y., Najjar, S. and Hudaib, M. (2010) A Validated RP HPLC-PAD Method for the Determination of Hederacoside C in Ivy-Thyme Cough Syrup. International Journal of Analytical Chemistry, 2010, Article ID: 478143, 5 p.

[4] Herbal Formulation Comprising Extracts of Adhatoda, Hedychium and Curcuma as Cough Syrup, WIPO Patent Application (2005) WO/2005/077393 Kind Code: A1.

[5] Saha, A., Pawar, V.M. and Jayaraman, S. (2012) Characterisation of Polyphenols in Terminalia arjuna Bark Extract. Indian Journal of Pharmaceutical Sciences, 74, 339-347.

[6] Said, H.M. (1970) Emblica Officinalis. In: Hamdard Pharmacopoeia of Eastern Medicine, The Times Press, Karachi, 383.

[7] Jacob, A., Pandey, M., Kapoor, S. and Saroja, R. (1988) Effect of the Indian Gooseberry (Amla) on Serum Cholesterol Levels in Men Aged 35 - 55 Years. European Journal of Clinical Nutrition, 42, 939-944.

[8] Mathur, R., Sharma, A., Dixit, V.P. and Varma, M. (1996) Hypolipidaemic Effect of Fruit Juice of Emblica officinalis in Cholesterol-Fed Rabbits. Journal of Ethnopharmacology, 50, 61-68. http://dx.doi.org/10.1016/0378-8741(95)01308-3

[9] Kim, H.J., Yokozawa, T., Kim, H.Y., Tohda, C., Rao, T.P. and Juneja, L.R. (2005) Influence of Amla (Emblica officinalis Gaertn.) on Hypercholesterolemia and Lipid Peroxidation in Cholesterol-Fed Rats. Journal of Nutritional Science and Vitaminology, 51, 413-418.

[10] Anila, L. and Vijayalakshmi, N.R. (2002) Flavonoids from Emblica officinalis and Mangifera indica-Effectiveness for Dyslipidemia. Journal of Ethnopharmacology, 79, 81-87. http://dx.doi.org/10.1016/S0378-8741(01)00361-0

[11] Rajak, S., Banerjee, S.K., Sood, S., Dinda, A.K., Gupta, Y.K., Gupta, S.K. and Maulik, S.K. (2004) Emblica officinalis Causes Myocardial Adaptation and Protects against Oxidative Stress in Ischemic-Reperfusion Injury in Rats. Phytotherapy Research, 18, 54-60. http://dx.doi.org/10.1002/ptr.1367

[12] Liu, L., Li, B. and Wang, L. (2005) Effect of Emblica officinalis on Formation of Atherosclerotic Plaque in Hypercholesterolemia Rabbits. Applied Journal of General Practice, 2, 97-98.

[13] Qureshi, S.A., Asad, W. and Sultana, V. (2009) The Effect of Phyllanthus Emblica Linn on Type-II Diabetes, Triglycerides and Liver-Specific Enzyme. Pakistan Journal of Nutrition, 8, 125-128. http://dx.doi.org/10.3923/pjn.2009.125.128

[14] Mand, J.K., Soni, J.L., Gupta, P.P. and Singh, R. (1991) Effect of Amla (Ebmlica officinalis) on the Development of Artherosclerosis in Hypocholesterolemic Rabbits. Journal of Research and Education in Indian Medicine, 10, 1-7.

[15] Pharmacopoeia Committee (2003) Monographs on Herbal Drugs of Unani Medicine. Drugs Control and Traditional Medicine Division, National Institute of Health, Islamabad, Vol. 1, 530.

[16] Kapoor, L.D. (1990) Ayurvedic Medicinal Plants. In: CRC Handbook of Ayurvedic Medicinal Plants, CRC Press, India, 322.

[17] Cheng, H.Y., Lin, T.C., Yu, K.H., Yang, C.M. and Lin, C.C. (2003) Antioxidant and Free Radical Scavenging Activities of Terminalia chebula. Biological \& Pharmaceutical Bulletin, 26, 1331-1335.

[18] Chattopadhyay, R.R. and Bhattacharyya, S.K. (2007) Plant Review Terminalia chebula: An Update. Pharmacognosy Reviews, 26, 1331-1335. 
Scientific Research Publishing (SCIRP) is one of the largest Open Access journal publishers. It is currently publishing more than 200 open access, online, peer-reviewed journals covering a wide range of academic disciplines. SCIRP serves the worldwide academic communities and contributes to the progress and application of science with its publication.

Other selected journals from SCIRP are listed as below. Submit your manuscript to us via either submit@scirp.org or Online Submission Portal.
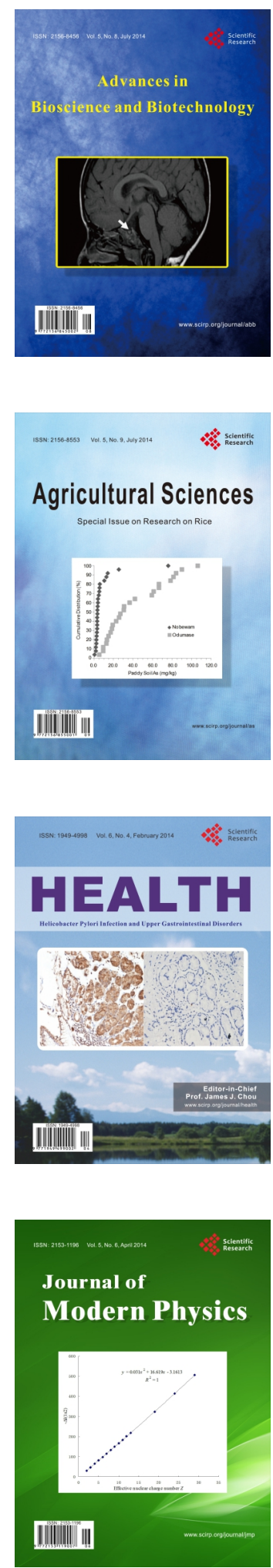
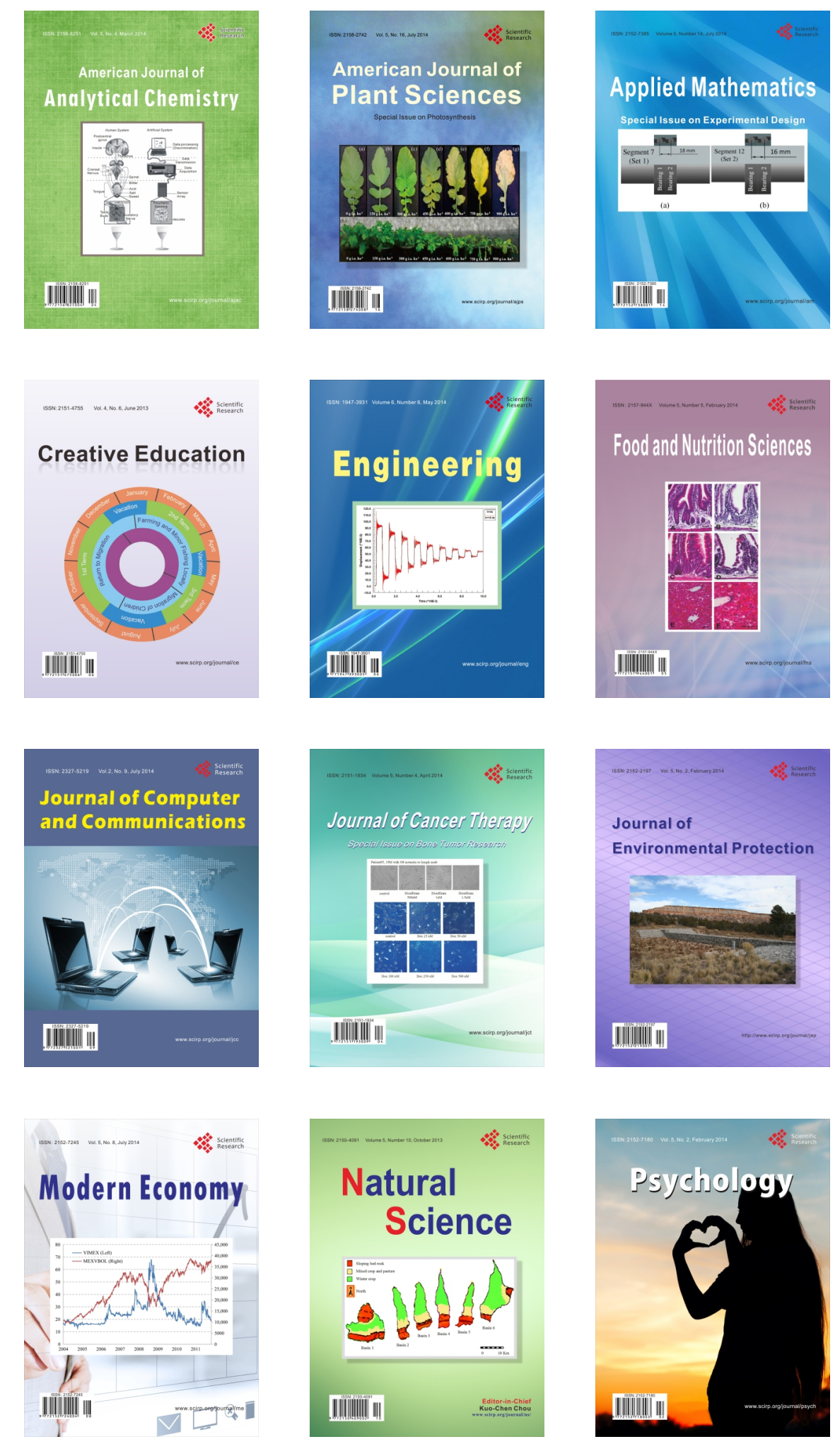